\title{
To Sweep or Not to Sweep
}

\author{
Olle Ekberg ${ }^{1}$
}

Received: 26 December 2018 / Accepted: 31 December 2018 / Published online: 7 January 2019

(c) Springer Science+Business Media, LLC, part of Springer Nature 2019

In this issue, Stephanie Watts et al. advocates to add an "esophageal sweep" in patients evaluated with modified barium swallow for evaluation of dysphagia. The aim of the paper is given in the abstract: to develop a cursory view of esophageal bolus flow. The approach may seem eclectic but miss the goal. Their suggestion enhances the current prejudice among radiologists that SLPs see the esophagus as an auxiliary tube not worthy of a meticulous evaluation. The technique described for the sweep is to observe four boluses: solids, tablets, and liquids $\times 2$. They claim that their method is able to reveal anatomical as well as motor dysfunctions. However, they also clearly state that they have no clue about false negatives. This superfluous cursory approach to the esophagus is in contrast to their careful approach to the pharynx. In fact, radiologic evaluation of swallowing in patients with dysphagia is a tremendous resource that when correctly performed and interpreted allows for a correct diagnosis in the vast majority of these patients [1-3]. Another problem with the paper by Stephanie Watts et al. is the confusing mixture about the aim of the radiologic examination. Is it a diagnostic test or a therapeutic test? The two should be strictly separated. In their paper, it seems as if therapy is started before a correct diagnosis of dysfunction is established. Moreover, the diagnostic test focuses on explaining the patients' symptoms and focuses basically on movement of anatomical structures. In contrast, the therapeutic study focuses on altering either the bolus or the physiology by altering respiration and/or head positioning. Therefore, first diagnosis, then treatment. If one problem in dysphagia is the cursory approach by the SLPs, another is the neglect from radiologists. During the last decades, gastrointestinal radiology has benefitted tremendously from new cross-sectional imaging techniques such as CT and MR. This has shifted the focus from fluoroscopy. As a consequence, few radiology departments have a strong interest in what has been called luminal gastrointestinal radiology [4]. In a perfect world, all diagnostic swallowing studies should be performed by such luminal gastrointestinal radiologists. When that is done SLPs, and maybe also other professionals with special training in radiologic evaluation, may perform the therapeutic examination in order to triage how to achieve the best effective and safe swallowing in a particular patient. In fact, the European Society for Swallowing Disorders will next year launch an initiative in order to streamline the performance and interpretation of radiology in this setting: ESSD Accreditation Programme for Videofluoroscopic Assessment for Oropharyngeal Dysphagia. However, this will not include the evaluation of the esophagus.

In conclusion: Do not rely on the cursory's sweep for evaluation of morphology and function of the esophagus.

\section{References}

1. Pokieser P, Scharitzer M. The clinical and radiological approach to dysphagia. In: Ekberg O, editor. Dysphagia. Diagnosis and treatment. 2nd ed. New York: Springer; 2018. p. 317-32.

2. Schima W, et al. Esophagus: radiologic evaluation of esophageal function. In: Ekberg O, editor. Dysphagia. Diagnosis and treatment. 2nd ed. New York: Springer; 2018. p. 451-76.

3. Levine MS, Rubesin SE. Diseases of the esophagus: a pattern approach. Abdom Radiol (NY). 2017;42:2199-218.

4. Levine MS, Trenkner SW. Training the next generation in luminal gastrointestinal radiology: a call to arms. AMJ. 2011;196:362-6.

Olle Ekberg $\mathrm{MD}, \mathrm{PhD}$
Olle Ekberg

olle.ekberg@med.lu.se

1 Division of Medical Radiology, Department of Translational Medicine, Skåne University Hospital, Lund University, Inga Marie Nilssons gata 49, 20502 Malmö, Sweden 